Ashdin Publishing

Journal of Generalized Lie Theory and Applications

Vol. 4 (2010), Article ID G100201, 8 pages

doi:10.4303/jglta/G100201

\title{
A formula for the number of Gelfand-Zetlin patterns
}

H. REFAGHAT ${ }^{a, b}$ and M. SHAHRYARI ${ }^{b}$

${ }^{a}$ Department of Mathematics, Islamic Azad University (Tabriz Branch), Tabriz, Iran

${ }^{b}$ Department of Pure Mathematics, Faculty of Mathematical Sciences, University of Tabriz, Tabriz, Iran

E-mails:refagat@tabrizu.ac.ir,mshahryari@tabrizu.ac.ir

\begin{abstract}
In this article, we give a formula for the number of Gelfand-Zetlin patterns, using dimensions of the symmetry classes of tensors.
\end{abstract}

2000 MSC: 15A69, 20C15

\section{Introduction}

Suppose $\Lambda=\left(a_{1}, a_{2}, \ldots, a_{n-1}\right)$ is a sequence of decreasing non-negative integers. A GelfandZetlin pattern based on $\Lambda$ is an array of integers:

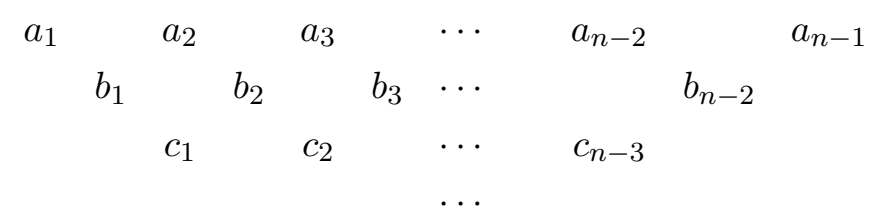

such that for all $i$,

$$
\begin{aligned}
& a_{i} \geq b_{i} \geq a_{i+1} \\
& b_{i} \geq c_{i} \geq b_{i+1}
\end{aligned}
$$

We denote the set of all Gelfand-Zetlin patterns based on $\Lambda$ by $\Gamma_{\Lambda}$. The set $\Gamma_{\Lambda}$ has an important role in the representation theory of general (equivalently, special) and orthogonal linear Lie algebras. For example, let $\Lambda$ be a dominant weight for the Lie algebra $\mathfrak{s l}_{n}(\mathbb{C})$ and suppose $L(\Lambda)$ is the corresponding irreducible representation with the highest weight $\Lambda$. In [5], Gelfand and Zetlin have proved that the set $\Gamma_{\Lambda}$ can be viewed as a basis for $L(\Lambda)$. For the matrix representations of the elements of the Chevalley basis of $\mathfrak{s l}_{n}(\mathbb{C})$ with respect to $\Gamma_{\Lambda}$, see [1] or [3]. The set $\Gamma_{\Lambda}$ is also important from the point of view of branching rules. Branching rules are descriptions of the reduction of irreducible representations upon restriction to a subalgebra (subgroup). The first branching rule discovered is possibly the well-known branching rule of representations of the symmetric group $S_{m}$ in the early twenties. 
Since then, it was an exciting job to discover other kinds of branching rules for finite groups, Lie groups, and Lie algebras. We can employ the set $\Gamma_{\Lambda}$ to describe the branching rule for type $\mathfrak{s l}_{n}(\mathbb{C}) \rightarrow \mathfrak{s l}_{r}(\mathbb{C})$. Suppose we like to restrict the representation $L(\Lambda)$ to $\mathfrak{s l}_{r}(\mathbb{C})$. For any Gelfand-Zetlin pattern $M \in \Gamma_{\Lambda}$, let $M^{i}$ be the $i$-th row of $M$. Then, the restriction of $L(\Lambda)$ to $\mathfrak{s l}_{r}(\mathbb{C})$ is equal to the following direct sum decomposition:

$$
\bigoplus_{M \in \Gamma_{\Lambda}} \frac{1}{\left|\Gamma_{M^{n-r+1}}\right|} L\left(M^{n-r+1}\right) .
$$

The aim of this article is to compute the number of elements of $\Gamma_{\Lambda}$. Although, one can use the well-known dimension formula of Weyl, but our formula is an alternative one, which uses the irreducible characters of the symmetric group. To give a survey of our main result, suppose

$$
m=a_{1}+2 a_{2}+3 a_{3}+\cdots+(n-1) a_{n-1} .
$$

We consider a partition $\pi$ of $m$ with the parts:

$$
\pi_{i}=a_{i}+a_{i+1}+\cdots+a_{n-1} .
$$

Let $\chi_{\pi}$ be the irreducible character of the symmetric group $S_{m}$ corresponding to $\pi$, (for standard terms about partitions and characters of $S_{m}$, see [11]). Also, for any permutation $\sigma \in S_{m}$, let $c(\sigma)$ be the number of disjoint cycles in the cycle decomposition of $\sigma$. It is clear that the function $\xi_{n}(\sigma)=n^{c(\sigma)}$ is a character of $S_{m}$, (for its irreducible constituents, see [12]). Our main result will be

$$
\left|\Gamma_{\Lambda}\right|=\left[\chi_{\pi}, \xi_{n}\right]
$$

where [, ] is the inner product of characters in $S_{m}$. In the other words, we will see that

$$
\left|\Gamma_{\Lambda}\right|=\frac{1}{m !} \sum_{\sigma \in S_{m}} \chi_{\pi}(\sigma) n^{c(\sigma)}
$$

\section{Symmetry classes of tensors}

In this section, we are going to review the notion of a symmetry class of tensors. The reader interested in the subject can find a detailed introduction in [9] or [10].

Let $V$ be an $n$-dimensional complex inner product space and let $G$ be a subgroup of the full symmetric group $S_{m}$. Let $V^{\otimes m}$ denote the tensor product of $m$ copies of $V$ and for any $\sigma \in G$, define the permutation operator:

$$
P_{\sigma}: V^{\otimes m} \longrightarrow V^{\otimes m}
$$

by

$$
P_{\sigma}\left(v_{1} \otimes v_{2} \otimes \cdots \otimes v_{m}\right)=v_{\sigma^{-1}(1)} \otimes v_{\sigma^{-1}(2)} \otimes \cdots \otimes v_{\sigma^{-1}(m)} .
$$

Suppose that $\chi$ is a complex irreducible character of $G$ and define the symmetrizer:

$$
S_{\chi}=\frac{\chi(1)}{|G|} \sum_{\sigma \in G} \chi(\sigma) P_{\sigma}
$$


The symmetry class of tensors associated with $G$ and $\chi$ is the image of $S_{\chi}$ and it is denoted by $V_{\chi}(G)$. So

$$
V_{\chi}(G)=S_{\chi}\left(V^{\otimes m}\right) .
$$

For example, if we let $G=S_{m}$ and $\chi=\varepsilon$, the alternating character, then we get $\wedge^{m} V$, the $m$-th Grassman space over $V$ and if $G=S_{m}$ and $\chi=1$, the principal character, then we obtain $V^{(m)}$, the $m$-th symmetric power of $V$, as symmetry classes of tensors.

Several monographs and articles have been published on symmetry classes of tensors during the last decades, see for example $[9,10]$.

Let $v_{1}, \ldots, v_{m}$ be arbitrary vectors in $V$ and define the decomposable symmetrized tensor:

$$
v_{1} * v_{2} * \cdots * v_{m}=S_{\chi}\left(v_{1} \otimes v_{2} \otimes \cdots \otimes v_{m}\right) .
$$

Let $\left\{e_{1}, \ldots, e_{n}\right\}$ be a basis of $V$, and suppose that $\Gamma_{n}^{m}$ is the set of all $m$-tuples of integers $\alpha=\left(\alpha_{1}, \ldots, \alpha_{m}\right)$ with $1 \leq \alpha_{i} \leq n$. For $\alpha=\left(\alpha_{1}, \ldots, \alpha_{m}\right) \in \Gamma_{n}^{m}$, we use the notation $e_{\alpha}^{*}$ for decomposable symmetrized tensor $e_{\alpha_{1}} * \cdots * e_{\alpha_{m}}$. It is clear that $V_{\chi}(G)$ is generated by all $e_{\alpha}^{*} ; \alpha \in \Gamma_{n}^{m}$. We define an action of $G$ on $\Gamma_{n}^{m}$ by

$$
\alpha^{\sigma}=\left(\alpha_{\sigma^{-1}(1)}, \ldots, \alpha_{\sigma^{-1}(m)}\right)
$$

for any $\sigma \in G$ and $\alpha \in \Gamma_{n}^{m}$. Given two elements $\alpha, \beta \in \Gamma_{n}^{m}$, we say that $\alpha \sim \beta$ if and only if $\alpha$ and $\beta$ lie in the same orbit. Suppose that $\Delta$ is a set of representatives of orbits of this action and let $G_{\alpha}$ denote the stabilizer subgroup of $\alpha$. Define

$$
\Omega=\left\{\alpha \in \Gamma_{n}^{m}:\left[\chi, 1_{G_{\alpha}}\right] \neq 0\right\},
$$

where [ , ] denotes the inner product of characters (see [7]). It is well known that $e_{\alpha}^{*} \neq 0$, if and only if $\alpha \in \Omega$, see for example [10]. Suppose $\bar{\Delta}=\Delta \cap \Omega$. For any $\alpha \in \bar{\Delta}$, we have the cyclic subspace:

$$
V_{\alpha}^{*}=\left\langle e_{\alpha^{\sigma}}^{*}: \sigma \in G\right\rangle \text {. }
$$

It is proved that we have the direct sum decomposition:

$$
V_{\chi}(G)=\sum_{\alpha \in \bar{\Delta}} V_{\alpha}^{*}
$$

see [10] for a proof. It is also proved that

$$
s_{\alpha}:=\operatorname{dim} V_{\alpha}^{*}=\chi(1)\left[\chi, 1_{G_{\alpha}}\right],
$$

and in particular, if $\chi$ is linear then $s_{\alpha}=1$ and so the set:

$$
\left\{e_{\alpha}^{*}: \alpha \in \bar{\Delta}\right\}
$$

is an orthogonal basis of $V_{\chi}(G)$. Also in the case of linear character $\chi$, we have $e_{\alpha^{\sigma}}^{*}=$ $\chi\left(\sigma^{-1}\right) e_{\alpha}^{*}$. In the general case, let $\alpha \in \bar{\Delta}$ and suppose

$$
e_{\alpha^{\sigma_{1}}}^{*}, e_{\alpha^{\sigma_{2}}}^{*}, \ldots, e_{\alpha^{\sigma_{t}}}^{*}
$$


is a basis of $V_{\alpha}^{*}$ with $\sigma_{1}=1$. Let

$$
A_{\alpha}=\left\{\alpha^{\sigma_{1}}, \alpha^{\sigma_{2}}, \ldots, \alpha^{\sigma_{t}}\right\} \text {. }
$$

Then, we define $\hat{\Delta}=\bigcup_{\alpha \in \bar{\Delta}} A_{\alpha}$. It is clear that

$$
\bar{\Delta} \subseteq \hat{\Delta} \subseteq \Omega
$$

and the set:

$$
\left\{e_{\alpha}^{*}: \alpha \in \hat{\Delta}\right\}
$$

is a basis of $V_{\chi}(G)$. Finally, we remember a formula for dimension of symmetry classes. We have

$$
\operatorname{dim} V_{\chi}(G)=\frac{\chi(1)}{|G|} \sum_{\sigma \in G} \chi(\sigma) n^{c(\sigma)}
$$

where $c(\sigma)$ denotes the number of disjoint cycles (including cycles of length one) in cycle decomposition of $\sigma$.

\section{$3 \quad$ Symmetry classes as $\mathfrak{s l}_{n}(\mathbb{C})$-modules}

In this section, we define a Lie module structure on $V_{\chi}(G)$, so let $L$ be a complex Lie algebra and suppose that $V$ is an $L$-module. For any $x \in L$, define

$$
D(x): V^{\otimes m} \longrightarrow V^{\otimes m}
$$

by

$$
D(x)\left(v_{1} \otimes v_{2} \otimes \cdots \otimes v_{m}\right)=\sum_{i=1}^{m} v_{1} \otimes \cdots \otimes x v_{i} \otimes \cdots \otimes v_{m} .
$$

We know that $D(x) S_{\chi}=S_{\chi} D(x)$ and so $V_{\chi}(G)$ is invariant under $D(x)$. Suppose

$$
D^{*}(x)=D(x) \downarrow_{V_{\chi}(G)},
$$

where the down arrow denotes restriction.

Definition 3.1. Define an action of Lie algebra $L$ on $V_{\chi}(G)$ by

$$
\begin{aligned}
x\left(v_{1} * \cdots * v_{m}\right) & =D^{*}(x)\left(v_{1} * \cdots * v_{m}\right) \\
& =\sum_{i=1}^{m} v_{1} * \cdots * x v_{i} * \cdots * v_{m} .
\end{aligned}
$$

Then, $V_{\chi}(G)$ becomes an $L$-module. In what follows, we will assume that $L=\mathfrak{s l}_{n}(\mathbb{C})$ and $V=\mathbb{C}^{n}$, the standard module for $L$. Then, $V_{\chi}(G)$ becomes an $L$-module. In [8], the irreducible constituents of $V_{\chi}(G)$ are determined. In this section, we give a summery of result of [8]. We also assume that $G=S_{m}$ and $\chi=\chi_{\pi}$, the irreducible character of $S_{m}$ corresponding a partition $\pi$. For simplicity, we denote the symmetry class of tensors by 
$V_{\pi}\left(S_{m}\right)$. To describe the irreducible constituents of $V_{\pi}\left(S_{m}\right)$, it is necessary to introduce some notations.

A Cartan subalgebra for $L$ is

$$
H=\left\{\operatorname{diag}\left(h_{1}, h_{2}, \ldots, h_{n}\right): h_{1}+h_{2}+\cdots+h_{n}=0\right\} .
$$

For any $1 \leq i \leq n$, define a linear functional:

$$
\mu_{i}: H \longrightarrow \mathbb{C}
$$

by

$$
\mu_{i}(h)=h_{i},
$$

where $h=\operatorname{diag}\left(h_{1}, h_{2}, \ldots, h_{n}\right)$, so we have

$$
\mu_{1}+\mu_{2}+\cdots+\mu_{n}=0
$$

and hence $\mu_{1}, \mu_{2}, \ldots, \mu_{n-1}$ is a basis for $H^{*}$.

Now let $\Lambda_{1}, \Lambda_{2}, \ldots, \Lambda_{n-1}$ be the fundamental weights corresponding to $H$. It is easy to see that for any $k$ :

$$
\Lambda_{k}=\mu_{1}+\mu_{2}+\cdots+\mu_{k} .
$$

Let $\alpha \in \Gamma_{n}^{m}$. We define a composition of $m$ by $m(\alpha)=\left(m_{1}, m_{2}, \ldots, m_{n}\right)$, where $m_{i}$ is the multiplicity of $i$ in $\alpha$. Suppose

$$
\mu_{\alpha}=\mu_{\alpha_{1}}+\mu_{\alpha_{2}}+\cdots+\mu_{\alpha_{m}} .
$$

So we have

$$
\mu_{\alpha}=m_{1} \mu_{1}+m_{2} \mu_{2}+\cdots+m_{n} \mu_{n} .
$$

Also we can see that

$$
\mu_{\alpha}=\left(m_{1}-m_{2}\right) \Lambda_{1}+\left(m_{2}-m_{3}\right) \Lambda_{2}+\cdots+\left(m_{n-1}-m_{n}\right) \Lambda_{n-1} .
$$

It is easy to prove that $\mu_{\alpha}=\mu_{\beta}$, if and only if $m(\alpha)=m(\beta)$. So, for any $\alpha \in \Gamma_{n}^{m}$, we introduce a partition $M(\alpha)$, which is just the multiplicity composition $m(\alpha)$ with a descending arrangement of entries. In fact, any partition of $m$, with height at most $n$, is of the form $M(\alpha)$, where $\alpha \in \bar{\Delta}$. For any $h \in H$ and $\alpha \in \hat{\Delta}$, we have

$$
h \cdot e_{\alpha}^{*}=\left(\sum_{i=1}^{m} \mu_{\alpha_{i}}\right)(h) e_{\alpha}^{*} .
$$

In other words, we have

$$
h \cdot e_{\alpha}^{*}=\mu_{\alpha}(h) e_{\alpha}^{*} .
$$

So the set of weights of $V_{\pi}\left(S_{m}\right)$ is $\left\{\mu_{\alpha}: \alpha \in \hat{\Delta}\right\}$. We also can see by an easy argument that the weight $\mu_{\alpha}$ is dominant, if and only if $M(\alpha)=m(\alpha)$, i.e. $m(\alpha)$ is a partition. For two dominant weights $\mu_{\alpha}$ and $\mu_{\beta}$, it is routine to check that $\mu_{\beta}$ appears in $L\left(\mu_{\alpha}\right)$ as a weight, if and only if $m(\alpha)$ majorizes $m(\beta)$, (for definition of the majorization, see [11]). We are now ready to compute irreducible constituents of $V_{\pi}\left(S_{m}\right)$. Although, the following theorem is proved in [8] in a more general framework, we prove it here again because what we need is only this special case. 
Theorem 3.2. We have

$$
V_{\pi}\left(S_{m}\right)=L\left(\mu_{\alpha}\right)^{\chi_{\pi}(1)},
$$

where $\alpha \in \bar{\Delta}$ is any element with the property $M(\alpha)=\pi$.

Proof. First of all, note that

$$
\bar{\Delta}=\left\{\alpha \in G_{n}^{m}: M(\alpha) \unlhd \pi\right\},
$$

where $G_{n}^{m}$ denotes the set of all increasing sequences in $\Gamma_{n}^{m}$ and $\unlhd$ denotes majorization. Suppose that $\eta_{1}, \ldots, \eta_{s}$ is the set of all dominant weights of $V_{\pi}\left(S_{m}\right)$, ordered in such a way that $\eta_{j} \preceq \eta_{i}$ implies $i \leq j$ (we say $\eta_{j} \preceq \eta_{i}$ iff $\eta_{i}-\eta_{j}$ is a sum of positive roots). As we saw above, for any $i$, there is $\alpha \in \bar{\Delta}$ such that $\eta_{i}=\mu_{\alpha}$. Suppose that $r_{i}$ equals number of $\beta \in \hat{\Delta}$ such that $m(\alpha)=m(\beta)$. Let $m_{i j}$ be the multiplicity of $\eta_{i}$ in $\eta_{j}$. Define a sequence of integers as follows:

$$
\begin{aligned}
& c_{1}=r_{1}, \\
& c_{i}=r_{i}-\sum_{j=1}^{i-1} m_{i j} c_{j} .
\end{aligned}
$$

Now we have

$$
V_{\pi}\left(S_{m}\right)=\sum_{i} L\left(\eta_{i}\right)^{c_{i}}
$$

so we must show that $c_{1}=\chi_{\pi}(1)$ and $c_{i}=0$ for $i \neq 1$. First, note that if $\alpha \in \bar{\Delta}$ has the property $\mu_{\alpha}=\eta_{1}$, then $m(\alpha)=\pi$ and hence we have

$$
\begin{aligned}
c_{1} & =r_{1} \\
& =|\{\beta \in \hat{\Delta}: m(\alpha)=m(\beta)\}| \\
& =|\{\beta \in \hat{\Delta}: \alpha \sim \beta\}| \\
& =s_{\alpha} \\
& =\chi_{\pi}(1)\left[1_{\left(S_{m}\right)_{\alpha}}, \chi_{\pi}\right] \\
& =\chi_{\pi} K_{\pi, m(\alpha)} \\
& =\chi_{\pi} K_{\pi, \pi} \\
& =\chi_{\pi}(1) .
\end{aligned}
$$

Note that $K_{*, *}$ denote the well-known Kostka numbers, see [11] or [4] for definition. Now, we compute $c_{2}$; we have $c_{2}=r_{2}-m_{2,1} c_{1}$. As in the above case, we easily see that $r_{2}=$ $\chi_{\pi}(1) K_{\pi, m\left(\alpha^{\prime}\right)}$, where $\alpha^{\prime}$ corresponds to $\eta_{2}$. It is proved that (see [4])

$$
K_{\pi, m\left(\alpha^{\prime}\right)}=m_{2,1} .
$$

Hence,

$$
\begin{aligned}
c_{2} & =\chi_{\pi} K_{\pi, m\left(\alpha^{\prime}\right)}-K_{\pi, m\left(\alpha^{\prime}\right)} \chi_{\pi}(1) \\
& =0 .
\end{aligned}
$$

By a similar argument, we see that $c_{i}=0$ for other " $i$ "s and the result follows. 
Note that this theorem affords a new method of constructing of all irreducible $\mathfrak{s l}_{n}(\mathbb{C})$ modules, namely, let $\Lambda=\mu_{\pi}$ be any integral dominant weight of $\mathfrak{s l}_{n}(\mathbb{C})$. As in [10], we have

$$
V_{\pi}\left(S_{m}\right)=\sum_{i=1}^{\chi_{\pi}(1)} V_{\pi}^{i}\left(S_{m}\right),
$$

where $V_{\pi}^{i}\left(S_{m}\right)$ is defined as follows: let

$$
F: S_{m} \longrightarrow G L_{\chi_{\pi}(1)}(\mathbb{C})
$$

be the corresponding representation of $\chi_{\pi}$ with $F(\sigma)=\left[a_{i j}(\sigma)\right]$. We introduce the partial symmetrizer $S_{\pi}^{i}$ by

$$
S_{\pi}^{i}=\frac{\chi(1)}{m !} \sum_{\sigma \in S_{m}} a_{i i}(\sigma) P_{\sigma} .
$$

Now, $V_{\pi}^{i}\left(S_{m}\right)$ is precisely the image of $S_{\pi}^{i}$. So we have

$$
L(\Lambda)=V_{\pi}^{i}\left(S_{m}\right)
$$

for all $1 \leq i \leq \chi_{\pi}(1)$. One of the most important consequences of this construction is the following dimension formula, which is different from Weyl's one.

Corollary 3.3. Let $L(\Lambda)$ be an irreducible $\mathfrak{s l}_{n}(\mathbb{C})$-module with the highest weight $\Lambda$ and define the corresponding number $m$ and the partition $\pi$ as in Section 1. Then,

$$
\operatorname{dim} L(\Lambda)=\frac{1}{m !} \sum_{\sigma \in S_{m}} \chi_{\pi}(\sigma) n^{c(\sigma)} .
$$

\section{The number of the Gelfand-Zetlin patterns}

We are ready now to give the interesting relation between dimension of the symmetry classes of tensors $V_{\pi}\left(S_{m}\right)$ and the number of Gelfand-Zetlin patterns based on $\Lambda$. Note that we have the following relations between the weight $\Lambda$ and the partition $\pi$ :

$$
\begin{aligned}
& m=a_{1}+2 a_{2}+3 a_{3}+\cdots+(n-1) a_{n-1}, \\
& \pi_{i}=a_{i}+a_{i+1}+\cdots+a_{n-1}, \\
& a_{i}=\pi_{i}-\pi_{i+1} .
\end{aligned}
$$

Main theorem. The number of Gelfand-Zetlin patterns based on $\Lambda$ is equal to the inner product $\left[\chi_{\pi}, \xi_{n}\right]$. Equivalently, we have

$$
\operatorname{dim} V_{\pi}\left(S_{m}\right)=\chi_{\pi}(1)\left|\Gamma_{\Lambda}\right| .
$$

Remark 4.1. In [12], the inner product $\left[\chi_{\pi}, \xi_{n}\right]$ is expressed in term of Kostka numbers. Suppose that $\rho=\left[\rho_{1}, \ldots, \rho_{s}\right]$ is a partition of $m$ with distinct parts $b_{1}, \ldots, b_{l}$. Suppose that the multiplicity of $b_{i}$ in $\rho$ is $r_{i}$. If $s \leq n$, define

$$
f(n, \rho)=\frac{n !}{(n-s) ! r_{1} ! r_{2} ! \cdots r_{l} !},
$$


and let $f(n, \rho)=0$ for $s>n$. Then the multiplicity of $\chi_{\pi}$ in $\xi_{n}$ is equal to

$$
\sum_{\rho} f(n, \rho) K_{\pi, \rho},
$$

where $K_{\pi, \rho}$ is the Kostka number.

Remark 4.2. Note that we can normalize $\Lambda$ in such a way that we have $a_{n-1}=1$. To do this, let $d=a_{n-1}-1$. Define

$$
\Lambda^{*}=\left(a_{1}-d, a_{2}-d, \ldots, a_{n-2}-d, 1\right) .
$$

Although in general $L(\Lambda)$ and $L\left(\Lambda^{*}\right)$ are non-isomorphic representations, it is clear that $\left|\Gamma_{\Lambda}\right|=\left|\Gamma_{\Lambda^{*}}\right|$. For $\Lambda^{*}$, we have

$$
m^{*}=m-\frac{n(n-1)}{2}\left(a_{n-1}-1\right),
$$

and also the corresponding partition $\pi^{*}$ has the parts:

$$
\pi_{i}^{*}=\pi_{i}-(n-1)\left(a_{n-1}+1\right) .
$$

Hence, we have also the following normalized formula for the number of Gelfand-Zetlin patterns:

$$
\left|\Gamma_{\Lambda}\right|=\left[\chi_{\pi^{*}}, \xi_{n}^{*}\right] .
$$

\section{References}

[1] A. O. Barut and R. Raczka. Theory of Group Representations and Applications. 2nd ed. PWNPolish Scientific Publishers, Warszawa, 1980.

[2] R. Carter. Lie Algebras of Finite and Affine Type. Cambridge Studies in Advanced Mathematics, 96, Cambridge University Press, Cambridge, 2005.

[3] L. Frappat, A. Sciarrino and P. Sorba. Dictionary on Lie Algebras and Superalgebras. Academic Press, San Diego, CA, 2000.

[4] W. Fulton and J. Harris. Representation Theory. A First Course. Graduate Texts in Mathematics, 129, Springer-Verlag, New York, 1991.

[5] I. M. Gelfand and M. L. Zetlin. Finite dimensional representations of a group of unimodular matrices. Dukl. Akad. Nauk. SSSR, 71 (1950), 825-828.

[6] J. Humphreys. Introduction to Lie Algebras and Representation Theory. Graduate Texts in Mathematics, 9, Springer-Verlag, New York, 1972.

[7] M. Isaacs. Character Theory of Finite Groups. Academic Press, New York, 1976.

[8] A. R. Madadi and M. Shahryari. Symmetry classes of tensors as $\mathfrak{s l}_{n}(\mathbb{C})$-modules. Linear and Multilinear Algebra, 56 (2008), 517-541.

[9] M. Marcus. Finite Dimensional Multilinear Algebra, Part I. Pure and Applied Mathematics, 23, Marcel Dekker, New York, 1973.

[10] R. Merris. Multilinear Algebra. Algebra, Logic and Applications, 8, Gordon and Breach Science Publishers, Amsterdam, 1997.

[11] B. Sagan. The Symmetric Group. Representation, Combinatorial Algorithms, and Symmetric Functions. The Wadsworth \& Brooks/Cole Mathematics Series, Wadsworth \& Brooks/Cole Advanced Books \& Software, Pacific Grove, CA, 1991.

[12] M. Shahryari and M. A. Shahabi. On a permutation character of $S_{m}$. Linear and Multilinear Algebra, 44 (1998), 45-52.

Received February 12, 2010

Revised April 26, 2010 